\title{
Enseñanza e investigación, un contrapunto posible \\ Una experiencia en Planeamiento en el área de la información
}

\author{
Yanet Fuster y Djamila Romani Parada \\ Facultad de Información y Comunicación. Universidad de la República. (FIC-UdelaR), Uruguay / \\ yanet.fuster@fic.edu.uy | https://orcid.org/oooo-0oo2-7447-8643/ \\ djamilaromani@fic.edu.uy | https://orcid.org/oooo-0oo1-9609-6833
}

\begin{abstract}
Resumen
La experiencia de evaluación de los aprendizajes, mediante la aplicación de un parcial procesual en la unidad curricular Planeamiento en el área de la Información, nos interpela en torno a los vínculos entre enseñanza e investigación y en cuanto al rol docente en el ámbito universitario como articulador de las tres funciones: enseñanza, investigación y extensión. En este recorrido proponemos un acercamiento al trabajo desarrollado en el aula sobre qué se planifica en unidades de información tomando como muestra algunas unidades de información de Montevideo.
\end{abstract}

\begin{abstract}
Teaching and research, a possible counterpoint: The experience in Planning in the area of information. The experience of evaluation of the learning through the application of a partial process in the curricular unit Planning in the area of Information questions us about the links between teaching and research and the role of teachers in the university field as an articulator of the three functions: teaching, research and extension. In this journey, we propose an approach to the work developed in the classroom on what is planned in information units taking as sample some units of information of Montevideo.
\end{abstract}

Artículo recibido: 26-10-2017. Aceptado: 10-03-2018

Palabras clave

\section{Enseñanza} Investigación Evaluación Aprendizaje Plan estratégico

Keywords

Teaching Research Evaluation

Learning

Strategic plan

\section{El punto de partida}

La propuesta que presentamos ha sido desarrollada en el marco de la unidad curricular Planeamiento en el área de la Información. Es un curso de grado que se dicta en el séptimo semestre de las Licenciaturas en Archivología y Bibliotecología en la Facultad de Información y Comunicación (FIC-UdelaR) en Uruguay. Se trata de una 
1. Como señaláramos en una publicación anterior, "nos referimos a la teoría psicológica del desarrollo cognitivo generado en el aula planteada primeramente por David Ausubel $(1973,1976$, 2002) y luego retomada por otros teóricos (Pozo, 1989; Moreira, 2000; Novak, 1998, entre otros), quienes también plantean la importancia del proceso según el cual se vincula una información nueva a la estructura cognitiva del que aprende. Esto ocurre de forma deliberada y atribuyendo significado a lo aprendido en la medida en que se interactúa con un universo que para el aprendiz resulta

relevante" (Fuster, 2015: 174). actividad de aula orientada a la observación en el ámbito de desempeño profesional y posterior análisis de temas tratados teóricamente en el curso, los cuales tienen como tópico nucleador el planeamiento y la gestión en las organizaciones. Tomamos una muestra aleatoria de unidades de información existentes en Montevideo con el objetivo de estimular la realización de intervenciones que permitan al estudiante una iniciación a la investigación. Para esto pusimos en diálogo los conceptos enseñados en la unidad curricular y las herramientas metodológicas que los estudiantes ya han aprendido en otros cursos de las carreras de Archivología y Bibliotecología.

El objetivo de esta contribución es plantear otra mirada acerca de los procesos de enseñanza, basados en la aplicación de estrategias para el aprendizaje significativo ${ }^{1}$. Respecto a la actividad propiamente dicha los objetivos que nos guiaron fueron los siguientes:

» Poner en diálogo el marco teórico transversal estudiado en el curso y los tópicos vinculados al módulo de planeamiento operativo (vinculado a la realización de planes y proyectos) a un caso práctico. Buscamos que el estudiante sea capaz de demostrar a través de esa integración: comprensión de los temas trabajados, capacidad de análisis y aplicación de los lineamientos teóricos sobre gestión de unidades de información a la realidad evidenciada en el ejercicio profesional.

» Integrar al dictado del curso nociones sobre prácticas letradas en el ámbito académico, en el entendido de que forman parte de la enseñanza de los contenidos disciplinares. En el caso que nos ocupa buscamos que el estudiante desarrolle estrategias para argumentar y fundamentar el recorrido propuesto en la tarea a realizar, con énfasis en el análisis de los resultados, ya sea en cuanto al desarrollo de los hallazgos presentados por escrito, así como también en la exposición oral ante sus pares y las docentes.

» Promover el desarrollo del pensamiento crítico a través de la articulación entre los aspectos teóricos programáticos del curso y la realidad evidenciada en la práctica profesional, al igual que en el análisis de los datos obtenidos mediante la aplicación de herramientas de investigación. También se busca que el estudiante sea capaz de trascender las palabras consignadas en los documentos y en las entrevistas e interpretarlas de acuerdo a la coyuntura analizada.

» Estimular el inicio temprano a la producción de trabajos de corte investigativo, con una metodología estipulada en relación con la forma y con cierta rigurosidad académica en cuanto a los contenidos, articulando este proceso con la reflexión sobre el rol del investigador en Ciencia de la Información.

\section{Desarrollo de la propuesta}

Dentro de los contenidos del curso se plantean una serie de temas que integran el módulo introductorio del programa de la unidad curricular, los cuales son concebidos como transversales, ya que si bien se presentan al comienzo del curso, a lo largo de todo el planteo conceptual se van efectuando anclajes hacia ellos y a partir de ellos: Sociedad de la Información y cultura posmoderna (identificación de los cambios globales que configuran los escenarios donde surgen los nuevos estilos de gestión); cultura organizacional; gestión de la información y el conocimiento; paradigmas societales y profesionales; enfoque sistémico y teoría de la complejidad integran el conjunto de esos tópicos concebidos como transversales. Buscamos poner en tensión los contenidos, hacer que los temas no se vean como compartimentos estancos para que el estudiante pueda ir incorporando conocimientos y vincularlos entre sí. En virtud de este planteo no existen bloques de conocimiento cerrado, pues a medida que se avanza en los contenidos del curso el estudiante necesita ir tejiendo la red de conexiones que le permitan ir avanzando en su trayecto dentro de la unidad curricular 
y también hacia otras unidades que ya han trabajado estos temas que nosotras retomamos en nuestro curso.

La consigna consistió en elegir al menos dos de estos temas transversales e identificar cómo se los visualiza en la práctica. El análisis de estos temas situados en el ámbito del ejercicio profesional se llevó a cabo a través de la visita y posterior realización de entrevistas por parte de los estudiantes a actores involucrados en la gestión de un servicio de información. La propuesta involucró una gama variada de unidades de información: bibliotecas, archivos, museos y otros espacios donde se desarrollan actividades vinculadas al tratamiento y difusión de información. Estos espacios fueron relevados por las docentes y luego de un proceso de selección integraron una lista que fue presentada ante los estudiantes para que eligieran en cuál de ellos intervenir.

En cuanto a la selección de la muestra, y por tratarse de una aproximación a la investigación, en el marco del curso, decidimos utilizar un muestreo no probabilístico, a través de una selección de casos "accidentales o por comodidad", en el que se "toman los casos o unidades que están disponibles en un momento dado" (Pineda; Luz y Canales de, 1994: 119). A su vez fue "intencional" y "deliberado", de acuerdo con Pineda, Luz y Canales de (1994) "el investigador decide, según los objetivos, los elementos que integrarán la muestra, considerando aquellas unidades supuestamente "típicas" de la población que se desea conocer." En este caso, la selección fue intencional, ya que tuvimos en cuenta un criterio más cercano a lo didáctico, considerando en la elección aquellos entornos profesionales pasibles de estudio y de que estuvieran de acuerdo con aplicación de la metodología de trabajo propuesta. A su vez debían ser espacios que brindaran variedad en cuanto a: modelos de administración, colecciones y cultura institucional, para sacar más provecho al análisis en relación con los temas transversales. Las docentes responsables del curso se contactaron con estas instituciones y plantearon la invitación a participar a quienes estuvieran dispuestos a recibir estudiantes y aceptar ser entrevistados, así como también aquellos dispuestos a compartir documentos institucionales donde se dieran evidencias de realización de algún tipo de planificación.

Así listamos las siguientes unidades de información entre el período 2016-2017

"Servicio de Documentación, Biblioteca y Archivo del BSE (Banco de Seguros del Estado).

»Centro de Documentación del Museo del Carnaval

" Centro de Documentación del Centro de Formación y Estudios del INAU (Instituto Nacional del Menor).

" Biblioteca del Banco República Oriental del Uruguay

» Biblioteca de ANCAP (Administración Nacional de Combustibles, Alcohol y Portland)

» Archivo de Imágenes de la UTE (Administración Nacional de Usinas y Transmisiones Eléctricas)

\subsection{Etapas del parcial procesual llevado adelante por los estudiantes}

Fases de la propuesta de evaluación:

» Fase 1: Producción de un texto argumentativo donde el estudiante presentó la institución elegida. Para ello sostuvo las razones que lo llevaron a inclinarse por la opción efectuada. Asimismo, debió establecer cuáles de los temas transversales pretendía analizar. Si bien los estudiantes optaron por al menos dos de estos temas transversales, en el transcurso del trabajo podían elegir otros si en el proceso se evidenciaba esa necesidad. Partimos del supuesto de que estas 
variantes surgirían mas allá de los temas elegidos, en el desarrollo de la visita a las instituciones, o en las entrevistas que los estudiantes realizarían en la unidad de información elegida pues, como ya dijimos anteriormente, concebimos el desarrollo de los contenidos en contrapunto, a lo largo y a lo ancho de todo el curso y no como compartimentos estancos.

"Fase 2: Una vez que las docentes efectuamos la devolución de la primera entrega y aceptamos la propuesta de análisis de los temas transversales elegidos a la luz del caso práctico, el equipo de estudiantes comenzó a desarrollar el trabajo en base a la pauta que planteamos para organizar la experiencia didáctica.

" Fase 3: En esta fase los estudiantes desarrollaron el esquema de entrevista que efectuarían a los responsables del servicio que estaban analizando, en concordancia con los temas transversales elegidos. Las docentes realizamos la devolución con sugerencias de ajustes en relación con los aspectos que los estudiantes pretendían indagar a través de las preguntas.

»Fase 4: Presentación y defensa del trabajo desarrollado a lo largo del semestre. En dicha instancia los estudiantes debieron detenerse en aquellos puntos que las docentes les sugerimos como oportunidades de mejora en las correcciones expuestas en las entregas anteriores (en esto precisamente se basa el carácter de procesual que posee el parcial, ya que en el proceso se va revisando el recorrido hecho y trabajando sobre los errores como oportunidades de mejora). La aprobación estuvo en concordancia al proceso hecho por el estudiante y a la defensa realizada a partir de su trabajo.

\subsection{Diseño de la entrevista}

Tomando como punto de partida los conocimientos incorporados en unidades curriculares vinculadas a metodología de la investigación, destinamos algunas clases a diseñar la entrevista y cada grupo la expuso ante los demás estudiantes en el espacio del aula. El trabajo se orientó en una primera instancia a efectuar un mapa de ruta a partir de los temas transversales en los que se focalizaría cada trabajo, y partiendo de ese abordaje se realizó el diseño de la modalidad de entrevista. Esta dinámica se realizó en forma personalizada con cada equipo de estudiantes.

\section{Características de las entrevistas}

a) Exploratorias.

En ellas los estudiantes se aproximaron al caso con la finalidad de obtener insumos para realizar luego una entrevista más profunda.

b) En profundidad y semiestructuradas.

Concebimos a la entrevista en profundidad como "encuentros estos, dirigidos hacia la comprensión de las perspectivas que tienen los informantes respecto de sus vidas, experiencias o situaciones, tal como las expresan con sus propias palabras. Las entrevistas en profundidad siguen el modelo de una conversación entre iguales, y no de un intercambio formal de preguntas y respuestas" (Taylor y Bogdan, 1992: 101).

El trabajo se realizó en equipos de hasta cinco estudiantes y contó con cuatro entregas a lo largo del semestre donde se fue avanzando en la complejidad del análisis, a partir de la guía docente. Fue presentado como una prueba parcial de carácter procesual, donde los estudiantes iban dando evidencia de sus logros en el aprendizaje a medida que podían ir encontrando en la práctica los aspectos que le permitieron vincular el planteo teórico desarrollado en el curso con la intervención institucional elegida.

Debemos destacar que todo esto ocurrió como parte de un proceso de intercambio y reelaboración de las ideas en el aula, donde primó la reflexión sobre la utilización 
de la entrevista como herramienta metodológica para la investigación cualitativa. Marcamos la relevancia de todos los detalles a tener en cuenta en el diseño, en la construcción, durante el transcurso de la misma y en el posterior análisis e interpretación de los datos. Este proceso se canalizó hacia una propuesta de entrevista que pudiera arrojar la mayor cantidad de datos precisos y pasibles de análisis, de acuerdo a los objetivos que cada grupo se planteaba y a las características de cada lugar. También es interesante en este proceso la reflexión sobre el rol de la investigación en Ciencia de la Información y la relevancia en el conocimiento que habían adquirido en la formación de grado en cuanto a metodología de investigación, ahora aplicada a la presente unidad curricular.

\section{Implicancias desde el planteo didáctico}

Bajo esta mirada el estudiante es activo protagonista de su aprendizaje, ya que si bien la caja de herramientas teóricas forma parte del contenido propuesto por las docentes, el modo en que esas herramientas fueron aplicadas y combinadas nació del estudiante, el cual fue asistido por las docentes, y acompañado por su grupo de clase. La concepción de enseñanza que subyace tiene su sustento en la cognición situada (Brown; Collins y Duguid, 1989), pues esta forma de concebir el aprendizaje sostiene que la actividad en que se despliega y desarrolla el conocimiento no puede divorciarse del aprendizaje y la cognición y debe insertase en la práctica. Por ello se requiere que sea contextualizado, pues es producto de las situaciones en que se produce. Se parte de la premisa de que "el conocimiento es situado, es parte y producto de la actividad, el contexto y la cultura en que se desarrolla y utiliza" (Díaz Barriga, 2003: 1).

El papel activo que asumen los sujetos que participan es evidente y por ello, además de lo expuesto, se buscó en forma permanente acompañar al estudiante hacia un aprender involucrándose y hacia una reflexión en la acción, ya que ese es el foco de atención de nuestra propuesta. El contenido disciplinar es concebido como un medio y no como un fin en sí mismo, pues el aprendiz deberá atribuirle sentido a esos contenidos en función de las situaciones-problema que se le presenten; de esta forma "aprender consiste en un acto intelectivo, pero a la vez social, afectivo y de interacción en el seno de una comunidad de prácticas socioculturales" (Díaz Barriga y Barroso, 2014: 38).

Otro aspecto interesante a destacar es la concepción de la evaluación como un proceso inserto en el propio proceso de enseñanza, pues se trata de varias etapas de una misma prueba que gradualmente se va desarrollando en clase. A medida que el estudiante va incorporando capacidades para internalizar los contenidos del curso, esos avances se aplican en el caso de estudio que ha elegido. Luego de la entrega de cada fase del trabajo las docentes efectuamos una devolución a los estudiantes, donde a través de comentarios a lo largo del texto que ellos presentan, les sugerimos oportunidades de mejora para que en la entrega de la siguiente fase incorporen una reformulación de esos aspectos, los cuales además serán revisados en la defensa oral del trabajo.

\section{Hallazgos en relación con los temas vinculados a planificación en las unidades de información}

De acuerdo al diseño de esta investigación, la aproximación al estudio de caso ha ocupado un lugar central. Esto no quiere decir que podamos generalizar y extender los resultados obtenidos. Sería más oportuno pensar en la posibilidad que brinda esta herramienta de ser transferida a otras situaciones. En tal sentido coincidimos con los autores que se inclinan a hablar de transferibilidad y no de generalización en 
las investigaciones de corte cualitativo, incluido el estudio de caso (Maxwell, 1998). Sumado a ello consideramos que dado que esta actividad no puede considerarse una investigación, no podemos tomar los resultados obtenidos a partir de las entrevistas como elementos a considerar de forma contundente sino que solo los visualizamos como un acercamiento a la investigación por parte de los estudiantes.

Una investigación de estudio de caso trata exitosamente con una situación técnicamente distintiva en la cual hay muchas más variables de interés que datos observacionales; y como resultado, se basa en múltiples fuentes de evidencia, con datos que deben converger en un estilo de triangulación; y, también como resultado, se beneficia del desarrollo previo de proposiciones teóricas que guían la recolección y el análisis de datos (Yin, 1994: 13).

Tal como el autor lo plantea, la riqueza de esta modalidad investigativa radica en el hecho de poder vincular múltiples fuentes presentes en un contexto rico y complejo, donde se requiere del empleo de un conjunto de estrategias para la construcción de la evidencia empírica. Nuestro objetivo se orientó a que el estudiante fuera capaz de mirar críticamente los resultados de su trabajo y lograra poner en diálogo los conceptos teóricos con lo que efectivamente ocurre en los ámbitos objeto de análisis.

El caso es uno entre otros; es altamente descriptivo y según Sautú (2003: 81): "particularístico", pues está focalizado sobre una situación "aun cuando en su elección se tenga en cuenta que es un caso entre otros con los que comparte ciertos rasgos". Si bien varias de las unidades de información pertenecen a la órbita estatal, no por esto podemos decir que los datos relevados puedan aplicarse a otras realidades ya que los usuarios que asisten a cada una de esas unidades de información también poseen características distintivas respecto a los otros.

Como ya hemos manifestado fue clave el aporte de técnicas de investigación que habían sido abordadas en otras unidades curriculares, ahora aplicadas a un trabajo concreto; no obstante, como dijimos, la (falta de) rigurosidad en la aplicación de la herramienta no permite arrojar resultados que puedan tomarse de forma tajante. Aun así queremos compartir los hallazgos más salientes de esta actividad que transversalizó el trabajo en Planeamiento en el área de la información a lo largo de todo el semestre.

La mayoría de las instituciones relevadas cuentan con algún tipo de planificación que ha sido redactada en documentos de diverso tipo, pero estos emanan de la institución mayor de la cual la unidad de información forma parte. No se manifiesta autonomía a la hora de desarrollar una planificación propia, separada de los lineamientos de la institución que acoge al servicio de información. A esto se suma que las bibliotecas, archivos y servicios de información, en general, no solo no cuentan con una planificación específica, sino que no participan activamente en la elaboración estratégica de la organización mayor.

Existe en el trabajo cotidiano indicios de la importancia de la planificación, de hecho los entrevistados dan cuenta de ello en sus intervenciones, ya que muchos lo destacan en la entrevista concibiéndola como una herramienta para gestionar la incertidumbre; pero dado que los planes formales que están escritos tienden a lo operativo, no queda claro si la concepción estratégica de la planificación se visualiza como necesaria. Generalmente se la emparenta con planes a muy largo plazo y la mirada suele ser más en relación con los problemas del día a día y cómo buscar caminos para resolverlos en un horizonte temporal cercano. En algunos casos ven como impedimento para desarrollar un plan estratégico la falta de personal y también el desconocimiento de cómo realizarla. 
Las organizaciones visitadas han demostrado una amplia apertura e interés en estos temas, así como también entusiasmo y colaboración en la formación de los estudiantes, en tanto aplicación de técnicas de investigación en el estudio de sus servicios como "caso". Se han mostrado con amplitud y disposición a recibir a los estudiantes, lo cual evidencia culturas institucionales abiertas, que al menos piensan en la posibilidad de cambios y están dispuestas a mostrar su funcionamiento y en tal sentido se orientan en la necesidad de realizar planificaciones específicas. Al ser instituciones que desde el primer momento accedieron a realizar la entrevista y dieron el aval para que los estudiantes las visitaran, resultaba un escenario previsible.

Con alguna excepción la mayor parte de los centros de información no definen sus presupuestos ni son ordenadores de gasto, dependen de jerarquías para planificar y tomar decisiones en cuanto a las adquisiciones. En algunos casos los profesionales encargados definen técnicamente qué comprar, de acuerdo a las solicitudes de acceso a la información, tanto en soporte papel o digital; pero no intervienen en la elaboración de cada presupuesto anual y general del servicio, si no que este se adjudica en razón de lo que las organizaciones mayores consideran.

Los temas más recurrentes tratados por los estudiantes en sus trabajos fueron el desarrollo del contexto socio-histórico (institucional y en relación con las políticas de información que están en juego a nivel del país, incluso en algunos casos presentaron un devenir histórico con la institución objeto de estudio y su situación actual) y la indagación sobre aspectos vinculados a la planificación estratégica (existencia de misión, visión que no sólo aparezca en los papeles, referencias a fortalezas, debilidades, oportunidades y amenazas evidenciadas por la institución y que sean tomadas en cuenta para el desarrollo de intervenciones en el medio). Los temas transversales surgieron casi en su totalidad en algún punto de la entrevista; más allá del diseño, en el análisis se observó la implicancia de todos los temas abordados como transversales, lo que demuestra que los estudiantes pudieron integrarlos y ponerlos en diálogo. También quedó planteado en los trabajos que la teoría se evidencia muy superficialmente en la práctica, dado que no existen planes estratégicos que las unidades de información por sí mismas hayan efectuado para sostener las actividades que realizan.

\section{Reflexiones finales}

Esta experiencia de evaluación de los aprendizajes en torno a la aplicación de un parcial procesual nos ha interpelado en relación con los vínculos entre enseñanza e investigación y en cuanto al rol docente en el ámbito universitario como articulador de las funciones que la universidad desarrolla. Si bien este trabajo es simplemente un ejemplo e intentó retomar aspectos vinculados a metodología de la investigación desarrollados en otras unidades curriculares, la intención fue tomar el aula como un laboratorio donde teoría y práctica fueran interpeladas y puestas en tensión.

El objetivo general que nos propusimos se ha cumplido, pues hemos desarrollado estrategias para el aprendizaje significativo. El estudiante fue incorporando nueva información a partir de sus conocimientos previos, que a su vez se fueron enriqueciendo y retroalimentando a lo largo del curso. También estimulamos el pensamiento crítico articulando teoría y práctica junto al análisis y la interpretación de los datos obtenidos a partir de las entrevistas. Las evaluaciones de los estudiantes acerca de la aplicación de esta modalidad de evaluación, inserta en el aprendizaje, y que trasciende el afán de medición de saberes, fue vista como un aspecto positivo que les dejó la unidad curricular Planeamiento en el área de la información. Ha sido un aprendizaje que al menos desde nuestro lugar consideramos significativo, pues sobre esos 
contenidos el estudiante fue volviendo una y otra vez para de esta forma conectarlos con la nueva estructura de conocimientos que iban desarrollando.

Consideramos que para que el aprendizaje resulte significativo, los conceptos y las ideas que el alumno maneja deben ser estables y claros pues de esa forma la nueva información puede interactuar con los conocimientos previos, de ahí que sea crucial dejar los contenidos y los criterios de evaluación claramente explicitados, lo cual fue considerado en la propuesta de trabajo que presentamos.

Los objetivos orientados claramente hacia la enseñanza de los contenidos del curso fueron trabajados a lo largo del proceso que el estudiante iba efectuando en su trayecto, y en aquellas oportunidades en que no demostraron comprensión cabal de los mismos, hubo instancias de reformulación por parte de los estudiantes, y posibilidades de retroalimentación desde la propuesta docente.

Muchas veces compartimos con colegas los procedimientos metodológicos o los lineamientos para evaluar y no nos detenemos a pensar en que podríamos integrarlos al trabajo de aula, construyendo conjuntamente con los estudiantes parámetros más claros y consignas detalladas sobre lo que entendemos relevante a la hora de valorar el desarrollo de capacidades. Sostenemos que la evaluación debe estar integrada al proceso de enseñanza y en tal sentido debe implicar una continuidad con la forma en que se ha planteado el abordaje de la asignatura dentro del aula y no una sorpresa para el estudiante.

Estas páginas intentaron poner en palabras el proceso de reflexión que hemos realizado como docentes acerca de nuestras propias prácticas de aula. Entendemos que esto es necesario y que debe hacerse siempre, en todo momento, preguntándose para qué o qué se evalúa en términos de aprendizaje y, sobre todo, el grado de validez; es decir, para qué o para quién se busca enseñar y, posteriormente, evaluar es significativo en relación al contexto social en que el sujeto está inserto. Esto implica además establecer claramente el lugar desde el cual se mira el mundo y el quehacer docente $\mathrm{y}$, en tal sentido, hemos buscado en todo momento la congruencia de la evaluación en relación a cómo se ha ido construyendo el conocimiento a lo largo del curso.

Las actividades de enseñanza y los procesos que buscan generar aprendizajes en los estudiantes plantean un desafío que no se restringe a impartir los contenidos propios de la asignatura. Habilitar el pensamiento critico, hacer del salón de clase un escenario para la expresión de puntos de vista, lograr que el curso sea un laboratorio de experimentación de prácticas que interpelen el quehacer profesional no es tarea sencilla, si además se piensa en el contexto universitario donde a las funciones de enseñanza les sumamos la investigación y la extensión. Asumimos el desafío de ir más allá, de no buscar resultados a corto plazo, sino aquellos que el trayecto del estudiante irá modelando con el tiempo, pues siguiendo conceptos de Elliott (1994: 12) "el resultado de este proceso es evidentemente complejo, impredecible, divergente y solo detectable en su profundidad a largo plazo". 


\section{Q Referencias Bibliográficas}

"Ausubel, D. 1973. Algunos aspectos psicológicos de la estructura del conocimiento. En Ausubel, D., org. La educación y la estructura del conocimiento. Investigaciones sobre el proceso de aprendizaje y la naturaleza de las disciplinas que integran el currículum. Buenos Aires: Ateneo. p. 211- 239.

»Ausubel, D. 1976. Psicología educativa. Un punto de vista cognoscitivo. México: Trillas.

"Ausubel, D. 2002. Adquisición y retención del conocimiento. Una perspectiva cognitiva. Barcelona: Paidós.

»Brown, J.; A. Collins y P. Duguid. 1989. Situated cognition and the culture of learning. En Educational Researcher. Vol. 18, no. 1, 32-42. <http://people.ucsc. edu/ gwells/Files/Courses_Folder/ED\%20261\%20Papers/Situated\%20Cognition.pdf> [Consulta: 12 octubre 2017].

"Díaz Barriga, F. 2003. Cognición situada y estrategias para el aprendizaje significativo. En Revista Electrónica de Investigación Educativa. Vol. 5, no. 2. <http:// redie.ens.uabc.mx/volsno2/contenido-arceo.html> [Consulta: 30 abril 2016].

"Díaz Barriga, F. y R. Barroso. 2014. Diseño y validación de una propuesta de evaluación auténtica de competencias en un programa de formación de docentes de educación básica en México. En Perspectiva Educacional. Formación de Profesores. Vol. 53, no. 1, 36-56. <http://www.perspectivaeducacional.cl/index.

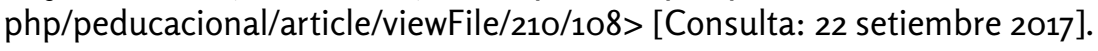

»Elliott, J. 1994. La investigación acción en la educación. Madrid: Morata.

» Fuster, Y. 2015. La evaluación integrada a la enseñanza - redireccionando una propuesta de parcial junto a los estudiantes de la Licenciatura en Bibliotecología. En Revista Bem Legal. Vol. 5, no. 2, 176-182. <http://www. paginas.ufrgs.br/ revistabemlegal> [Consulta: 5 abril 2018].

" Maxwell, J. 1998. Designing a Qualitative Study. En Bickman L. y D. J. Rog, eds. Handbook of Applied Social Research Method. Thousand Oaks, CA, Sage. p. 69-100.

》Moreira, M. 2000. Aprendizaje significativo: teoría y práctica. Madrid: Visor.

"Novak, D. 1998. Conocimiento y aprendizaje. Madrid: Alianza.

»Pineda, E.; E. Luz y F. Canales de. 1994. Metodología de la investigación. Manual para el desarrollo de personal de la salud. Washington: OMS.

»Pozo, J. 1989. Teorías cognitivas del aprendizaje. Madrid: Morata.

»Sautú, R. 2003. Todo es teoría. Objetivos y métodos de investigación. Buenos Aires: Lumiere.

» Taylor, S. y R. Bogdan. 1992. Introducción a los métodos cualitativos en investigación. La búsqueda de los significados. Madrid: Paidós.

" Yin, R. 1994. Case Study Research: Design and Methods. Thousand Oaks, CA: Sage Publications. 
